\title{
Investigación De Las Actitudes De Los Productores Ganaderos En La Implementación De Los Sistemas Silvopastoriles En El Cantón Joya De Los Sachas
}

\author{
Ing. Raúl Lorenzo González Marcillo, MSc \\ Carrera Zootecnia-Extensión Norte amazónica-Escuela Superior \\ Politécnica de Chimborazo, Riobamba-Ecuador
}

Doi: 10.19044/esj.2018.v14n27p357 URL:http://dx.doi.org/10.19044/esj.2018.v14n27p357

\begin{abstract}
This paper focuses on analyzing the level of implementation of silvopastoral options as part of livestock production systems in Joya de los Sachas, Orellana, Ecuador. The attitudes of producers about the silvopastoral systems and the factors that influence the decisions and actions related to the use of this type of production systems were explored. The research shows that the producers' decision-making process can be explained through the analysis of natural resource management problems. This was performed based on a theoretical framework of complex systems and classical institutional economics. In this research, both qualitative and quantitative methods were used in data collection and analysis. All the producers in the sample have positive attitudes toward the implementation of silvopastoral systems, which is proven by the existence of at least one silvopastoral option in their production systems. However, the levels of implementation show great variation among producers. The decisions of producers are explained by a combination of factors. In order to promote the implementation of this technology, it is important to have a dialogue with producers and understand their motivations. No biophysical factors were observed that seem to limit the level of implementation. This fact makes the potential of diversified silvopastoral systems very promising in livestock farms.
\end{abstract}

Keywords: Silvopastoral system, woody forage, pasture degradation, soil compaction, adoption of innovations

\section{Resumen}

El presente artículo analiza el nivel de implementación de opciones silvopastoriles como parte de los sistemas de producción ganadera en Joya de los Sachas, Orellana, Ecuador. Se exploraron las actitudes de los productores/as acerca de los sistemas silvopastoriles y los factores que 
influyen en las decisiones y acciones relacionadas con el uso de este tipo de sistemas de producción. La investigación muestra que el proceso de toma de decisiones de los productores se puede explicar mediante el análisis de problemas de manejo de recursos naturales, basado en un marco teórico de sistemas complejos y economía institucional clásica. En esta investigación se utilizaron tanto métodos cualitativos como cuantitativos en la toma y análisis de datos. Todos los productores en la muestra tienen actitudes positivas hacia la implementación de sistemas silvopastoriles, lo cual se comprueba con la existencia de al menos una opción silvopastoril en sus sistemas de producción. Sin embargo, los niveles de implementación presentan gran variación entre los productores. Las decisiones de los productores se explican por una combinación de factores, por lo que para promover la implementación de esta tecnología es importante tener un diálogo con los productores y entender sus motivaciones y el contexto en que éstas se dan. No se observaron factores biofísicos que parezcan limitar el nivel de implementación. Ese hecho hace que el potencial de los sistemas silvopastoriles diversificados sea muy prometedor en fincas ganaderas.

Palabras Clave: Sistema silvopastoril, leñosas forrajeras, degradación de pasturas, compactación de suelos, adopción de innovaciones

\section{Introduction}

El propósito de la investigación es conocer las actitudes que tienen los productores ganaderos con respecto al uso y manejo de los sistemas silvopastoriles, cuya meta es diversificar la producción y aumentar los beneficios sociales, económicos y ambientales de los sistemas de producción animal, sistemas en los cuales el ganadero introduce deliberadamente arboles maderables, frutales y leñosas forrajeras dentro de los pastizales y en cercas vivas. La degradación de pasturas tiene implicaciones importantes tanto ecológicas (p.e. reducción en la captura de $\mathrm{C}$, erosión y compactación de suelos, pérdida de biodiversidad), como sociales (p.e. mayores distancias recorridas con los animales) y económicas (menores ingresos por vaca y por hectárea). Las causas de la degradación de pasturas son muchas y muy complejas. Herty, et al. (2007) las agrupan en: (a) fallas en la selección de especies y en el establecimiento de pasturas; (b) manejo inadecuado de los pasturas, como puede ser la falta de fertilización o el uso inadecuado del fuego; (c) fallas en el manejo del pastoreo, como es el sobrepastoreo, o la falta de períodos adecuados de descanso; (d) factores bióticos, como la presencia de plagas y enfermedades; y (e) factores abióticos, como el exceso o déficit de lluvias, fallas en drenaje y baja fertilidad de los suelos. En el caso de América Central este problema se ha hecho más agudo por la movilización de la producción ganadera. Murgueitio, et al. (2003), indican que la solución son 
los sistemas silvopastoriles como una verdadera oportunidad para promover el desarrollo sostenible de grandes regiones rurales latinoamericanas que hoy se encuentran bajo usos ganaderos degradantes y de baja productividad. Los sistemas silvopastoriles surgen como respuesta a necesidades tales como: condición de suelos restrictivos, aumentar la producción de carne $\mathbf{y}$ leche e ir de la mano con el uso sustentable del medio ambiente ya que permite reducir la emisión de gas metano (participante en el efecto invernadero). De este modo, dichos sistemas resultan en una serie extra de beneficios para la actividad ganadera y para los productores que los implementen, además del atractivo retorno que presentan, ya que logran un adecuado balance entre productividad, estabilidad, diversidad y autorregulación del ambiente (UNED, 2012). Por esta razón, el principal objetivo de este estudio es determinar las actitudes de los productores ganaderos de la Joya de los Sachas, provincia de Orellana, con respecto a la implementación de sistemas silvopastoriles.

\section{Materiales y Métodos \\ Descripción del área de estudio}

El área de estudio abarcó los territorios y coordenadas de los Cantones Joya de los Sachas S $0.30014^{\circ}$ y O $76.85750^{\circ}$, perteneciente a la Provincia de Orellana, Ecuador. En el área de estudio existen dos estaciones climáticas predominantes, una tropical húmeda y otra tropical muy húmeda. Posee una estación marcada entre los meses de febrero a agosto, y una precipitación entre 2800 y $3000 \mathrm{~mm}$. La temperatura promedio anual oscila entre los $18^{\circ} \mathrm{C}$ y 32 ${ }^{\circ} \mathrm{C}$. La zona de estudio tiene igualmente una altitud promedio de $275 \mathrm{msnm}$ (INAMHI, 2010). Las principales actividades económicas que generan fuentes de trabajo en los municipios son la ganadería y la agricultura, en la que destacan los cultivos de maíz, arroz, café y cacao y palma africana.

\section{Marco de análisis y variables estudiadas}

Para el análisis se seleccionaron variables independientes medibles o que se pueden registrar dentro de cada categoría de factores (Conocimiento local y formación de experiencia, Actitudes acerca de las acciones, Control de acciones percibido, Característica del actor, Acciones, Atributo del recursoTecnología, Oportunidades y recursos requeridos, Instituciones: convencionales, normas y leyes formales). Las variables potenciales de cada categoría fueron identificadas a partir de estudios teóricos y empíricos (Dagang \& Nair, 2003; Pattanayak, et al., 2003; Shriar, 2001; Current, et al., 1995). La selección final se hizo a lo largo del trabajo de campo, lo que permitió evaluar las variables más relevantes en el contexto específico del área de estudio. Las variables independientes que se incluyeron en cada categoría fueron: 
Atributos del recurso y de la tecnología: tamaño del hato, edad promedio de los potreros, proporción del área de potreros con pastos mejorados, si produce leche o no, densidad de ganado con respecto al área de potreros (carga animal).

Características del productor y de su familia: nivel de educación del productor y de los miembros del grupo familiar, número de miembros de la familia que trabajan en la finca.

Instituciones sociales: manejo de los árboles, intercambio de lo aprendido de sus padres acerca de los árboles, participación en organizaciones sociales (cooperativa), posesión de título de propiedad, acceso al mercado, distancia al mismo, si tiene carro o no.

Conocimiento local, información y experiencia: número de años que el productor ha trabajado en su finca, número de fuentes formales de información acerca de árboles (programas de asistencia técnica u otras fuentes), número de puntos que el productor recibe según un índice de conocimiento sobre árboles.

Oportunidades y recursos necesarios: disponibilidad de tiempo para trabajar con sistemas silvopastoriles, acceso a asistencia técnica y financiamiento, acceso a semillas de árboles.

Mediante un análisis cualitativo se identificaron los factores que influyen en el proceso de toma de decisiones; para ello, se construyeron índices que cuantifican las actitudes y acciones. El índice que denota el nivel de implementación de sistemas silvopastoriles está conformado por los siguientes componentes: (i) la complejidad de los agroecosistemas y la generación de servicios ambientales, los cuales se cuantificaron con la metodología del proyecto GEF/Silvopastoril Murgueitio, et al. (2003); (ii) el cambio en la densidad de los árboles en los potreros durante los últimos 8 y 13 años; (iii) las consideraciones tomadas en cuenta para el manejo y el número de usos de productos de árboles reportados y observados. Este índice refleja las acciones de los productores.

El índice que evalúa las actitudes de los productores acerca de la implementación de sistemas silvopastoriles se basa en lo expresado por ellos en cuanto a los beneficios y desventajas de los árboles. Se aplicó un análisis de factores y de correlación para diseñar los índices y probar su sensibilidad. Los índices se usaron como variables dependientes en el análisis de regresión.

\section{Colección y análisis de datos}

Los datos del estudio provinieron de varias fuentes, entre ellas: a) datos existentes (una encuesta socioeconómica administrada a 17 productores); b) entrevistas estructuradas aplicadas en el campo (17); c) caminatas de con 
entrevistas semi-estructuradas; d) un grupo de discusión; e) entrevistas no estructuradas y observación participativa con productores ganaderos de la zona y f) entrevistas semi-estructuradas con cuatro organizaciones que trabajan en el cantón.

El análisis combina métodos cualitativos (grounded theory, coding y análisis de narrativas) y métodos cuantitativos (estadística descriptiva, análisis de correlación, regresión stepwise y análisis de factores). Todas las variables utilizadas en el análisis cualitativo se recopilaron usando parámetros estandarizados (de la encuesta socioeconómica, el mapeo del campo o entrevistas estructuradas).

\section{Resultados y discusión}

\section{¿Cuál es la actitud de los productores hacia los sistemas silvopastoriles?}

Los datos de 17 productores encuestados confirman que tienen algún elemento silvopastoril (árboles) disperso dentro de potreros, en divisiones de potreros (cercas vivas) y en sus sistemas de producción, el cual les reporta algún beneficio social, económico y/o ambiental. Sin embargo, los niveles de implementación y de beneficios recibidos presentan gran variación entre productores. Potencialmente, todos los productores podrían disfrutar de más beneficios que los que obtienen hoy en día. Un patrón interesante que se observó es que, salvo unas pocas excepciones, los productores solamente emplean los productos de los árboles para auto-consumo en el hogar y como factores de producción en la ganadería. Muy pocos aprovechan los productos de los árboles (frutos, resinas) con fines de comercialización.

Esta tendencia coincide con las observaciones de Shriar, (2001) en Joya de los Sachas y Francisco de Orellana, quien afirma que esto sucede por el bajo precio en el mercado para productos como café y cacao, y el alto riesgo de sembrar cultivos con altos costos de producción, como el arroz y maíz.

Hay una diferencia importante entre los sistemas de producción dirigidos a la comercialización, los que se limitan a la producción para autoconsumo y los que usan opciones silvopastoriles como factores de producción. Tales estrategias de producción tienen potencialidades importantes para aumentar los beneficios del productor, pero el contexto es el que determina la estrategia que sigue el productor y esto, en consecuencia, tiene un impacto sobre la manera en que se podrían promover los sistemas silvopastoriles. El presente estudio apunta a que la producción para comercialización de productos de árboles es relativamente insignificante. Por tanto, las iniciativas orientadas a promover la adopción de sistemas silvopastoriles deben tomar en cuenta las estrategias y el contexto dentro del cual trabajan los productores. El Banco Mundial discute lo mismo en su reporte acerca de los costos y beneficios de la adopción de opciones agroforestales en Centroamérica y el Caribe (Current, et al., 1995; World Bank, 1996). También Dagang \& Nair 
(2003) analizaron la importancia de considerar el contexto en que toma decisiones el productor.

Entre los beneficios derivados de los árboles que los productores mencionaron destacan, en primer lugar, productos que tienen una utilidad concreta (postes, madera o frutas), pero también mencionaron otros menos tangibles como la relación entre los árboles y la lluvia, la producción de oxígeno y el albergue y alimento para animales silvestres. Lo anterior demuestra que los productores también valoran los servicios ambientales que los árboles proveen.

Según lo expresado por la mayoría de los productores, sus actitudes (la manera en que valoran los sistemas silvopastoriles) corresponden a un nivel de adopción más alto de lo que se observa en la realidad de sus fincas. La mayoría de los productores prefieren las cercas vivas y bosque y árboles dispersos, pero los potreros típicos de los productores en la zona tienen árboles mucho más dispersos (menores densidades de árboles). Sólo cinco de los productores entrevistados consideran que tener árboles en los potreros tiene más desventajas que beneficios.

Más del $44 \%$ de los productores mencionaron los siguientes beneficios asociados con los árboles en potreros: sombra para los animales, postes para cercas muertas, forraje para los animales (frutas, semillas y hojas), cercas vivas, frutas para el consumo de la familia y algo de sombra para el pasto $\mathrm{y}$ animales (Figura 1).

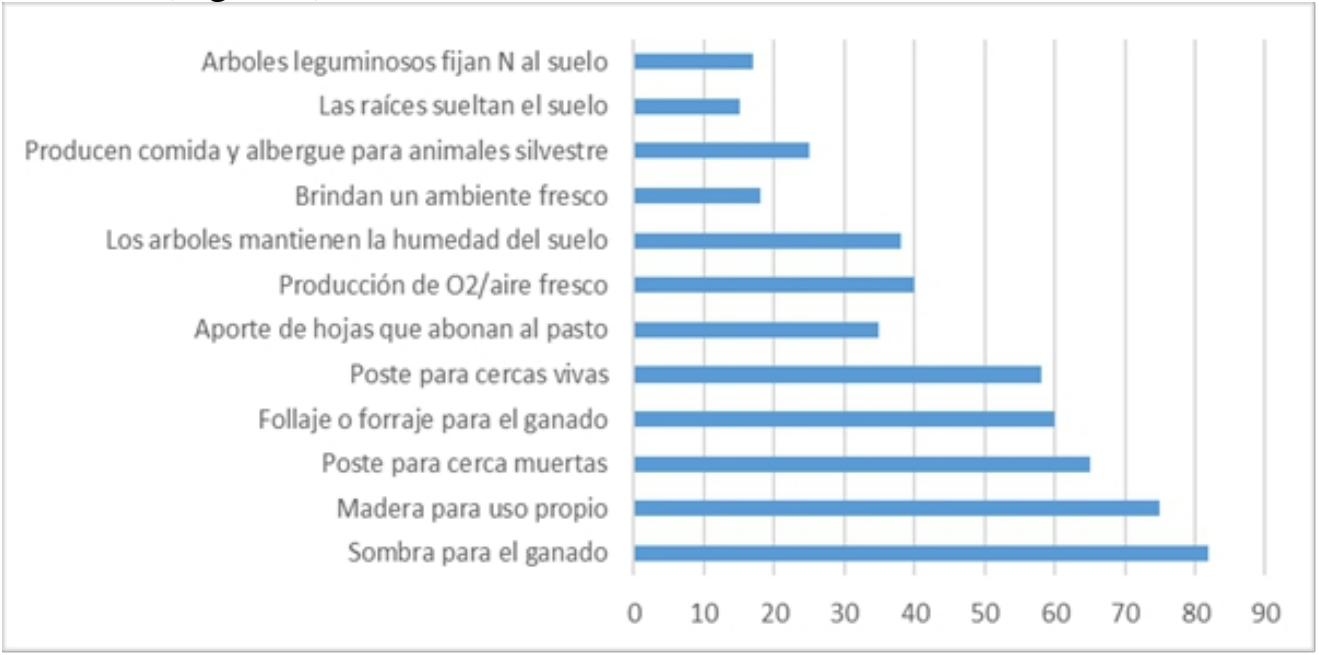

Figura 1. Beneficios de los árboles en potreros mencionados por los productores ganaderos.

La única desventaja mencionada por más del $24 \%$ de los productores es que demasiada sombra interfiere con el crecimiento del pasto (Figura 2). Entre los que mencionaron esa desventaja, muchos especificaron, sin embargo, que depende de la especie de árboles. Las caminatas en los 
transeptos confirmaron el alto conocimiento de los productores acerca de las diferentes especies de árboles y sus usos.

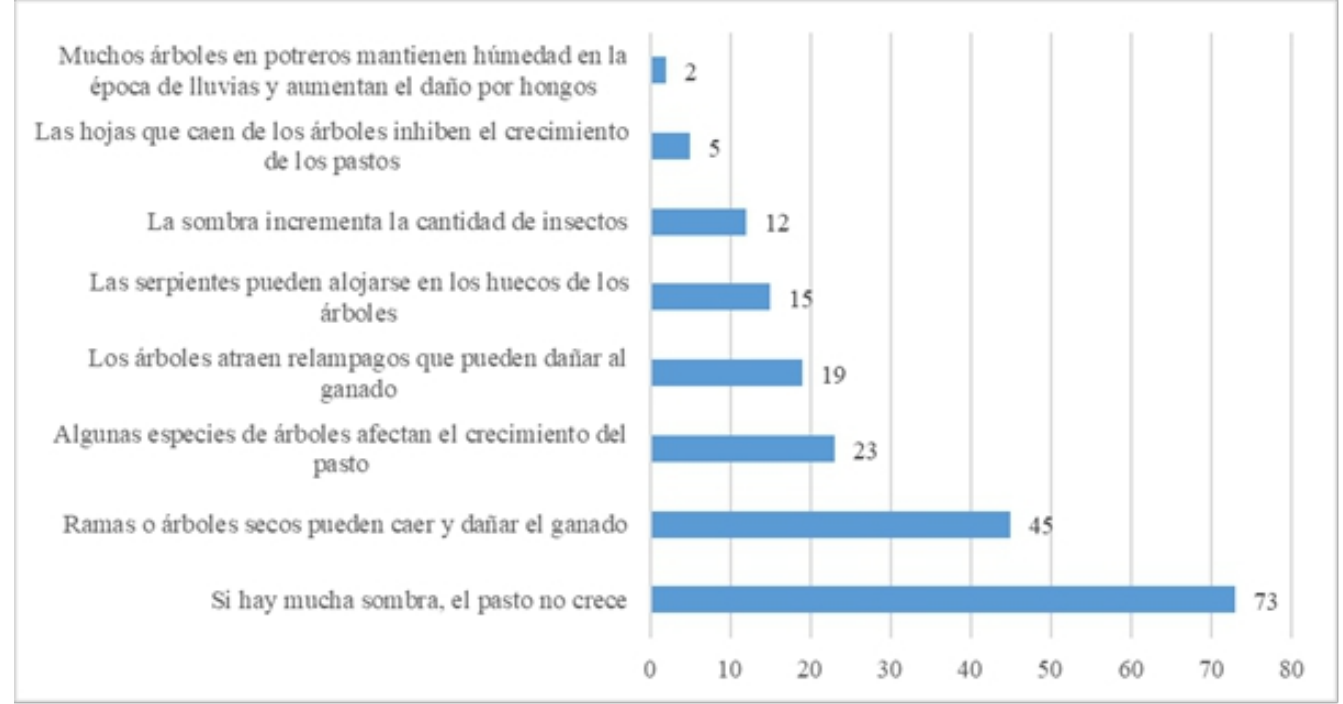

Figura 2. Desventajas de los árboles en potreros citadas por los productores Ganaderos.

\section{¿Qué factores influyen en la adopción de sistemas silvopastoriles por parte de los productores? ¿Qué factores son importantes para que las actitudes se conviertan en acciones?}

A continuación, se mencionan las variables que resultaron significativas en el análisis de regresión, con un nivel de confianza de 0,90. Hay variables de todas las categorías de factores que influyen directa o indirectamente sobre las actitudes y acciones; por eso, el marco de análisis puede ser útil para entender el proceso de toma de decisiones de los productores. Tal proceso es bastante complejo; no se puede encontrar una solución sencilla que explique por qué el nivel de implementación de sistemas silvopastoriles no es más alto. No hay una variable o una razón que explique la decisión de implementación de sistemas silvopastoriles. En este sentido, la recomendación de Pattanayak, et al. (2003) \& Shriar (2001) acerca de incluir un espectro amplio de variables en estudios de adopción de tecnologías agrícolas por parte de los productores es totalmente válida. Para promover la adopción de tecnologías es importante tener un diálogo con los productores y entender sus motivaciones y pensamientos, pues las decisiones de los productores se explican por una amplia combinación de factores.

Los resultados parecen indicar que algunas variables influyen en direcciones contrarias sobre las actitudes y las acciones implementadas por los productores. Algunas de estas contradicciones son fáciles de explicar. Por ejemplo, los resultados apuntan a que los productores con potreros más viejos 
tienen actitudes más negativas, pero acciones más favorables hacia la implementación de árboles en potreros. Los datos cualitativos indican que la manera más común de establecer un potrero nuevo en la zona es talar el bosque, cultivar maíz y arroz unos años y después establecer pasto o café y cacao y dejar que los árboles deseados crezcan. Esa tradición de manejo resulta en que los potreros jóvenes normalmente tienen muy pocos árboles, mientras los más viejos tienen la cantidad de árboles que el productor decidió dejar. Entonces, es lógico pensar que la edad de los potreros y las acciones de los productores se correlacionan positivamente. La correlación negativa entre la edad de los potreros y las actitudes se puede explicar, probablemente, con la relación que existe entre la edad de los potreros y la cantidad de árboles presentes en los mismos. Los productores con potreros jóvenes, donde todavía no hay la cantidad de árboles deseada, están anuentes a establecer más árboles. Una mayor parte de los productores con potreros viejos, en cambio, ya tienen la cantidad de árboles que ellos consideran ideal, aunque esta cantidad sea poca.

Otros cambios de dirección en la influencia entre actitudes y acciones son más difíciles de explicar. Es difícil sugerir una explicación lógica de por qué el hablar sobre el manejo de árboles con otros productores influye positivamente en las actitudes, pero negativamente en las acciones. Como el número de productores que fueron entrevistados es limitado $(n=17)$ se debe tener cuidado con las conclusiones que se derivan solo del análisis cuantitativo. A partir de análisis cuantitativo únicamente, es difícil concluir si un patrón observado realmente señala una relación causal, una relación no causal, o si es producto de casualidades estadísticas. Esa inseguridad hace necesaria la combinación de herramientas cuantitativas y cualitativas. La diferencia entre la influencia sobre las actitudes y las acciones enseña también que en el proceso de toma de decisiones es importante distinguir entre estos dos pasos. Un programa que busque alentar la implementación de sistemas silvopastoriles no necesariamente llegará a su meta si está basado sólo en información acerca de las actitudes de los productores.

\section{Conclusiones}

La conclusión más relevante de esta investigación es que los productores tienen actitudes positivas hacia la implementación de sistemas silvopastoriles y que todos pudieran recibir más beneficios al adoptar esa tecnología diversificada. Sin embargo, no se encontró ningún factor biofísico que tenga una influencia consecuente negativa en la implementación de sistemas silvopastoriles. En el grupo de factores de atributos del recurso y la tecnología, sólo el tamaño del hato parece correlacionarse negativamente con las acciones. Los datos no permitieron incluir variables acerca del tipo de suelo y el paisaje en el análisis cuantitativo, pero las observaciones en el campo y 
las entrevistas cualitativas parecen indicar que las tecnologías de sistemas silvopastoriles realmente tienen el potencial de ser implementadas bajo un gran espectro de condiciones biofísicas. Ese hecho hace que el potencial de adopción de sistemas silvopastoriles diversificados sea muy prometedor.

\section{References:}

1. Current, D., Lutz, E., \& Scherr, S. (1995). Costs, benefits, and farmer adoption of agroforestry: project experience in Central America and the Caribbean.. Washington DC, World Bank IFPRI-CATIE-UNDP. World Bank Environment Papers No. 14. Vol 1. 232

2. Dagang, ABK., \& Nair, PKR. (2003). Silvopastoral research and adoption in Central America: recent findings and recommendations for future directions. Agroforestry Systems 59:149-155.

3. Herty Betancourt, D.A., Pezo, J., Cruz, Y., Beer, J. (2007). Impacto Bioeconómico de la Degradación de Pasturas en Fincas de Doble Propósito en El Chal, Petén, Guatemala. Centro Agronómico Tropical de Investigación y Enseñanza (CATIE) Turrialba 7170, Costa Rica.

4. INAMHI (2010). Anuario Meteorológico. Versión Preliminar No. 50. Quito-Ecuador.

5. Murgueitio, E., Ibrahim, M., Ramírez, E., Zapata, A., Mejía, C., \& Casasola, F. (2003). Usos de la tierra en fincas ganaderas. Cali, CO, Fundación Centro para la Investigación en Sistemas Sostenibles de Producción Agropecuaria. 97 p.

6. Pattanayak, S.K., Mercer, E., Sills, E., \& Yang, J. C. (2003). Taking stock of agroforestry adoption studies. Agroforestry Systems 57:173186.

7. Shriar, A.J. (2001). The dynamics of agricultural intensification and resource conservation in the buffer zone of the Maya Biosphere Reserve, Petén, Guatemala. Human Ecology 29 (1):27-48.

8. UNED., UTPL., \& AIESAD (2012). Evaluación de un sistema institucional autorregulatorio para un ambiente en línea: el caso de psicología en México. vol. 15, num. 2.

9. World Bank (1996). Costs, benefits, and farmer adoption of agroforestry: project experience in Central America and the Caribbean. Washington DC, World Bank Enviro. 